\title{
Patient Positioning During Digital Rectal Examination of the Prostate: Preferences, Tolerability, and Results
}

\author{
Frederico R. Romero, Antonio W. Romero, Renato Tambara Filho, Thadeu Brenny Filho, \\ Fernando Cesar de Oliveira Júnior
}

Hospital de Clínicas da Universidade Federal do Paraná (FRR, TF, TBF); Instituto Curitiba de Saúde (FRR, FCO Jr); Hospital Policlínica Cascavel, PR, Brazil (FRR, AWR)

\begin{abstract}
Purpose: To evaluate the preferred position used by Brazilian Urologists to perform DRE, the position that Brazilian patients prefer or think it is less embarrassing to have a DRE, and to evaluate the results of DRE with patients in left lateral decubitus, modified lithotomy, standing-up, or the physician will have them place their elbows on the table and squat down slightly.

Materials and Methods: Brazilian Urologists were contacted by e-mail, and 200 patients answered a questionnaire while undergoing prostate cancer screening.

Results: The preferred position was modified lithotomy position reported by $63.4 \%$ of Urologists, and left lateral position reported by $42.7 \%$ of the patients. Total DRE time was lower in the standing-up position. Pain and urinary urgency scores were similar regardless of the position used, and bowel urgency score was higher in patients squatting down. Patients were similar in terms of age and PSA level, but there was a significant difference between the standard deviations of estimated prostate weight in left lateral position. There were no differences in prostate asymmetry, positive DRE, or incomplete palpation of the prostate rates among different examination positions.

Conclusions: Despite individual subjective preferences, a faster examination time in the standing-up position, and higher bowel urgency scores in patients with their elbows placed on the table and squatting down slightly, there were similar rates of prostate asymmetry, positive DRE, and incomplete palpation of the prostate, and comparable patient tolerability among different examination techniques.
\end{abstract}

Key words: patient positioning; prostate; diagnosis; pain; digital rectal examination

Int Braz J Urol. 2011; 37: 371-379

\section{INTRODUCTION}

Digital rectal examination (DRE) was the only diagnostic test for prostate cancer before 1920, and although diagnostic procedures have increased in number, complexity, and expense (1), DRE is still an important part of prostate cancer screening because of its simplicity, limited time and expenses, and relative little discomfort involved. Furthermore,
DRE may detect more clinically significant prostate cancers, decreasing rates of overdiagnosis and overtreatment of potentially indolent prostate cancers.

Several positions have been recommended over the years to allow a thorough DRE, from the exaggerated knee-chest position and bimanual sitting-rectal position commonly used in the past $(2,3)$, which are frequently uncomfortable, complicated and time-consuming, to the straightforward and most 
frequently used positions (lateral position, modified lithotomy position, and standing-up position) (4-6).

Although positioning preferences are individual and, there is little published information comparing different position techniques during prostate cancer screening (4-6). The purpose of this study is to evaluate the preferred position used by Brazilian Urologists to perform DRE, in which position Brazilian patients prefer or perceived as less embarrassing to undergo DRE.

\section{MATERIALS AND METHODS}

\section{Urologists' preferences}

Urologists from 4 Brazilian States (PR, RS, $\mathrm{SC}$, and SP) were contacted by e-mail regarding their preferences on patient positioning during DRE. Three e-mails were sent for all members of Brazilian Society of Urology in each State. The first email was entitled "Research on prostate cancer screening", and it had one question regarding in which position he/she usually performed DRE when screening for prostate cancer. The second and third emails were entitled "SECOND CALL" and "LAST CALL", respectively, and they were sent with 14 and 30 days delay between communications only for Urologists that did not answer the preceding e-mail. In the body of the e-mail, there was a figure illustrating the different positions, and a description of each possible alternative (Figure-1).

\section{Patients' preferences}

Two hundred patients undergoing prostate cancer screening answered if they had received DRE in the past. If 'yes', they answered, based on Figure-1, in which position they were examined previously, and in which position they preferred - or they perceived as less embarrassing - to undergo DRE. Patients who had no preference were examined in the same position they underwent DRE previously. If 'no', they responded in which position they preferred undergoing DRE for the first time. When no preference was stated, they were positioned at the discretion of the examiner.

\section{Tolerability}

Immediately after DRE, patients quantified the intensity of pain, urinary urgency, and urge to defecate felt during examination using a 0 -to-10 visual analog scale (VAS).

\section{Results}

Total DRE time (in seconds) was recorded starting after patient instructions were given, including removing the pants down to patient's knees level, patient positioning, DRE, and clothing. Time using the toilet after examination was not included.

Estimated prostate weight (in grams), prostate asymmetry, positive DRE (presence of hard nodules), and incomplete palpation of the prostate rates were calculated for each position.

All patients were examined by a single examiner with 5 years of clinical experience after a 4-year Residency in Urology.

Statistics

Categorical variables were analyzed using Fisher Exact Test. Continuous data were evaluated using t-Student Test. Statistical significance in this study was set as $\mathrm{p}<0.05$.

\section{RESULTS}

\section{Urologists' preferences}

We received $506(27.7 \%)$ answers from a total 1,827 e-mail contacts to Urologists from one Southeastern and three Southern Brazilian States; $193(10.6 \%)$ e-mails were undeliverable, and 1,128 (61.7\%) did not answer. The preferred position reported by 321 Urologists was modified lithotomy position (63.4\%), followed by left (or right) lateral position $(17.0 \%)$, standing-up position $(14.2 \%)$, squatting down with elbows on the table (3.4\%), and other position $(1.0 \%)$.

\section{Patients' preferences}

From a total 200 patients, $111(55.5 \%)$ had already received DRE in the past. In this group, when asked in which position they preferred - or 

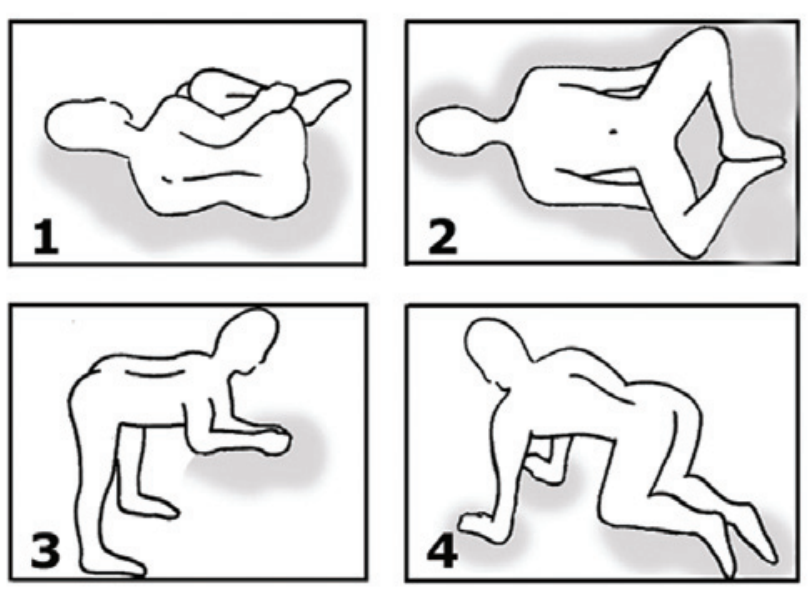

Figure 1 - The Burdizzo clamp (A) positioned to crush the spermatic cord (B).

thought it was less embarrassing - to undergo DRE, $69(62.2 \%)$ patients chose the same position used previously, $24(21.6 \%)$ chose a different position than in past DRE, and $18(16.2 \%)$ patients had no preference (Table-1). Thirty-eight (55.9\%) patients that had DRE in modified lithotomy position chose a different position in current DRE, and 2 patients who underwent examination in left lateral position or standing-up position chose a different position in current DRE (8.3\% and $13.3 \%$, respectively).

Eighty-nine (44.5\%) patients underwent DRE for the first time. The preferred position chosen by these patients was left lateral position
(42.7\%), and $15(16.9 \%)$ patients had no preference (Table-1). Overall, the preferred position was left lateral decubitus (37\%), followed by standing-up $(20.5 \%)$, modified lithotomy $(19.5 \%)$, and squatting down with elbows on the table $(6.5 \%)$.

\section{Tolerability}

The four groups of patients undergoing one of each position illustrated in Figure-1 were similar in terms of age and PSA level (Table-2). Pain, urinary urgency, and urge to defecate scores are summarized in Table-3. Urge to defecate score was higher in the squatting down position with elbows on the table, in comparison with the standing-up position. All other scores were comparable regardless of the position used.

\section{Results}

Total DRE time was lower for the standingup position compared to all other groups. Timing among modified lithotomy position, left lateral position, and squatting down with elbows on the table was similar. Estimated prostate weight was different in the left lateral position when compared to modified lithotomy position, and to squatting down with elbows on the table. There was no difference in prostate asymmetry, positive DRE, or incomplete palpation of the prostate rates among the different posi-

Table 1 - Patient positioning in past DRE, and patients' preferences in current DRE.

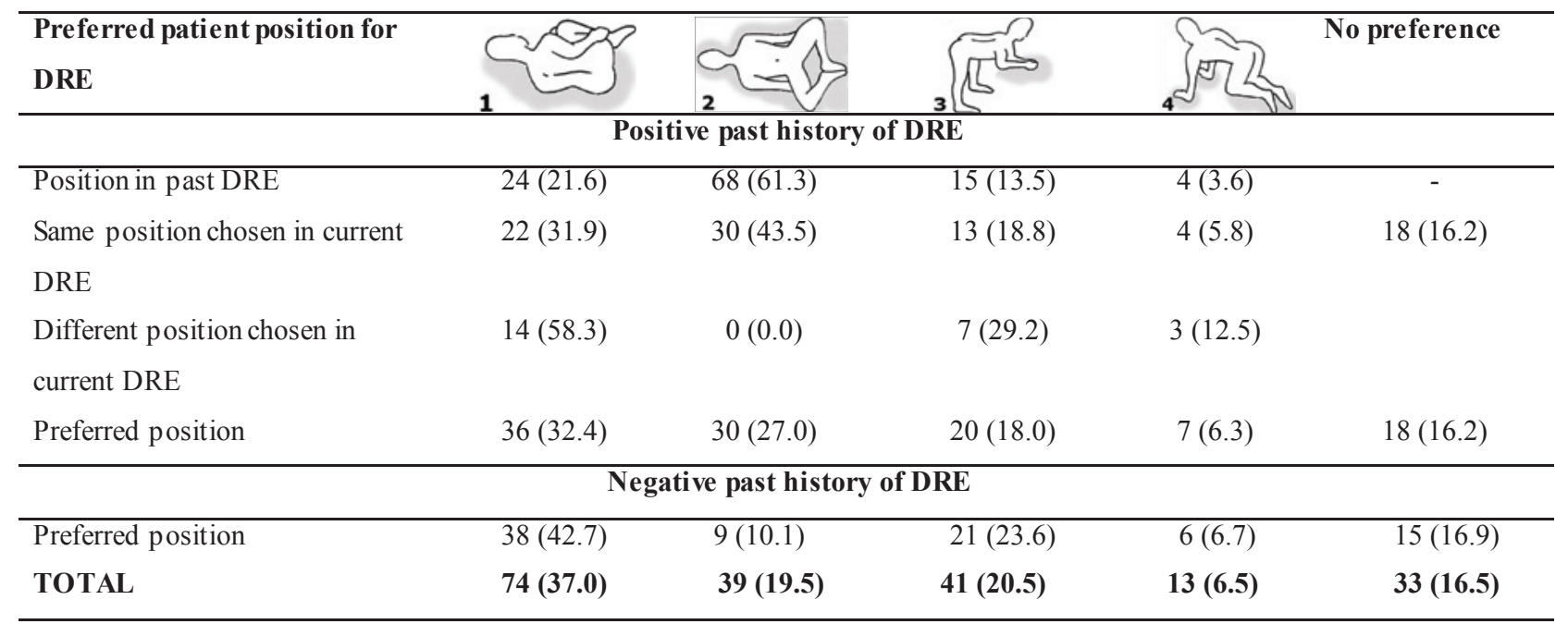


Table 2 - Age and PSA level of the patients.

\begin{tabular}{|c|c|c|c|c|c|}
\hline Pain/discomfort & & & & & p value \\
\hline \multirow[t]{2}{*}{ Age (years) } & $50.0 \pm 8.3$ & $51.0 \pm 7.3$ & $52.5 \pm 7.3$ & $55.0 \pm 8.7$ & $>0.05$ \\
\hline & $(39-77)$ & $(40-72)$ & $(41-71)$ & $(43-68)$ & \\
\hline \multirow[t]{2}{*}{ PS A (mg/dL) } & $0.97 \pm 1.7$ & $1.18 \pm 2.9$ & $1.20 \pm 2.6$ & $0.87 \pm 1.5$ & $>0.05$ \\
\hline & $(0.1-7.4)$ & $(0.1-17.1)$ & $(0.2-13.0)$ & $(0.3-5.0)$ & \\
\hline
\end{tabular}

tions (Table-4). The number of patients in each group was not large enough to allow comparison between rates of prostate cancer detection in each position. Two (1.0\%) patients had prostate cancer overall, both in the left lateral position group.

\section{DISCUSSION}

Positioning preference for examining the prostate varies from one country to another (4), from one examiner to another (5), and even from one patient to another (6). In the US, DRE is more often performed in the standing-up position, while in UK patients are usually examined in the lateral position (4). In Brazil, Messina et al. reported that the preferred position for DRE reported by 846 Urologists is the dorsal decubitus position (57.9\%) (5), similar to the modified lithotomy position re- ported in the present study, which was the preferred position claimed by $63.4 \%$ of responders.

Furlan et al. reported that $53.9 \%$ of 269 patients preferred DRE in supine position (similar to modified lithotomy position) (6). A potential bias in this study was that patients answered the question regarding their preference after receiving DRE in supine position. In the present study, we observed that patients' preferences are influenced by past experience, given that $62.2 \%$ of patients chose the same position used in past DRE. Therefore, patient preference may be to some degree related to examiner preference. The preferred position reported by Brazilian Urologists (modified lithotomy position) was validated by the fact that $61.3 \%$ patients receiving DRE in the past were examined in this position. Previous experience certainly influenced patients' preferences, since all

Table 3 - Patient tolerability of DRE.

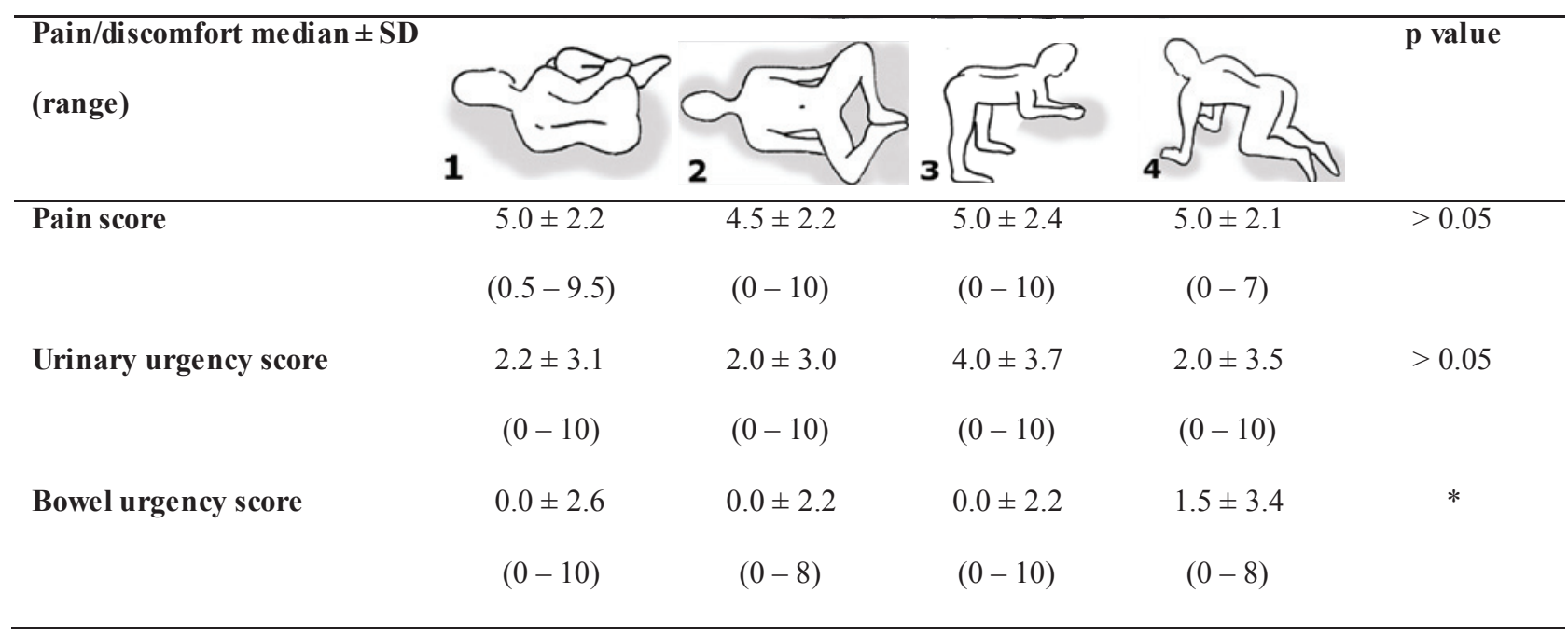


Table 4 - Results of DRE.

\begin{tabular}{|c|c|c|c|c|c|}
\hline Results & & & & & p value \\
\hline Total DRE times \pm & $140.5 \pm 27.6$ & $142.0 \pm 23.8$ & $111.0 \pm 18.6$ & $148.0 \pm 20.0$ & * \\
\hline SD (range) & $(93-241)$ & $(104-202)$ & $(86-168)$ & $(124-188)$ & \\
\hline Estimated prostate & $40 \pm 10.6$ & $40 \pm 12.6$ & $40 \pm 11.2$ & $45 \pm 8.1$ & ** \\
\hline weigth $g \pm S D($ range $)$ & $(20-80)$ & $(20-80)$ & $(20-70)$ & $(35-60)$ & \\
\hline $\begin{array}{l}\text { Prostate asymmetry } \\
\text { rate No. }(\%)\end{array}$ & $19(21.1)$ & $10(21.3)$ & $10(19.2)$ & $3(30.0)$ & $>0.05$ \\
\hline $\begin{array}{l}\text { Nodularity rate No. } \\
\text { (\%) }\end{array}$ & $2(2.2)$ & $2(4.2)$ & $2(3.8)$ & $0(0.0)$ & $>0.05$ \\
\hline $\begin{array}{l}\text { Incomplete palpation } \\
\text { of the prostate rate }\end{array}$ & $2(2.2)$ & $2(4.2)$ & $2(3.8)$ & $0(0.0)$ & $>0.05$ \\
\hline No. (\%) & & & & & \\
\hline
\end{tabular}

$* p<0.001$ in total DRE time for all groups compared to standing-up position

** $p<0.05$ in estimated prostate weigth between modified lithotomy position, and kneeling while resting on the table with the hands compared to left-lateral position

patients that chose modified lithotomy position in current DRE were positioned in the same way in previous DRE examinations. In Furlan's study, if patients were able to choose a different position than supine, most patients would have preferred the standing-up position, followed by left lateral position (6). In the present study, patients undergoing DRE for the first time picked left lateral position first and standing-up position second. In the group of patients that had received DRE in the past, the preferred position was, again, left lateral position, followed by modified lithotomy position and standing-up position. Based on these results, most patients prefer or perceive as less embarrassing to undergo DRE in the left lateral position or the standing-up position. In addition, we observed that the majority of patients that had DRE in the past have a tendency to be positioned in the same way, and that $16.5 \%$ of patients have no preference, or they believe the selection of positioning is up to the examiner. Only one study compared patient preference between standing-up position and left lateral position, and found better acceptance of the standing-up position (7).
Despite individual subjective preferences, there is little objective information to suggest that positioning of the patient makes much difference in the results of DRE. In the present study, we found that total DRE time was lower for the standing-up position. Since DRE was performed in all patients by one examiner in the same medical office, the single difference in total DRE time between each position was patient positioning itself. Lower DRE time in standing-up position may be due to needless laying on the examining table, and speedier clothing after examination. Although time should not be a major issue while evaluating patients' health, faster examination may be valuable if standing-up position prove to be as effective as other positions, particularly when examining large number of patients in prostate cancer screening programs.

Early in the past century, Frank Hinman stated that the exaggerated knee-chest position (on the knees with hips to heels and chest to knees) was the only position that allowed a thorough palpation of the prostate and seminal vesicles (2). Although some conditions such as obesity, hip 
osteoarthritis, bedridden patients, or extremely large prostates may require special positioning or bimanual examination to allow for complete palpation of the prostate (3), currently, the majority of patients may be satisfactorily examined in one of the four positions. Overall, we found a $0 \%$ to $4.2 \%$ rate of incomplete palpation of the prostate, similar between the different positions studied. When the prostate cannot be completely palpated, additional flexion of the hips and knees is a simple method that frequently allows a complete examination.

Subtle findings such as asymmetry between prostate lobes may be suggestive of the presence of prostate cancer, and considered by some to be an indication for prostate biopsy (8). Empirically, when prostate asymmetry is acknowledged in left lateral position, patients should be examined again in a different position to confirm asymmetry. This was not confirmed in the present study, since prostate asymmetry rate was similar between the different positioning techniques.

Romero et al. reported that pain and discomfort during DRE are not negligible, and that measures to decrease patient distress should be evaluated (9). In their study, in which all patients were positioned in modified lithotomy, $61 \%$ reported moderate to unbearable pain, and $22 \%$ reported moderate to unbearable urinary urgency or urge to defecate during DRE (9). Using a VAS, Furlan et al. reported a mean pain score of 1.68 for DRE in supine position (6), and Kaygisiz et al. found a mean pain score of 2.59 in left lateral position (10). In our study, mean and median pain scores were respectively 4.63 and $5.00 \pm 2.3$ for the entire cohort, similar among the different positions evaluated. Patients in standing-up position, however, had a tendency to higher urinary urgency score (median $4.00 \pm 3.7$ ). Urge to defecate score was higher in patients positioned squatting down with elbows on the table (median $1.50 \pm 3.4)$ in comparison with the standing-up position $(0.00 \pm 2.2)$. These results should be interpreted with caution due to the small number of patients.

Patients in the different subgroups of our study were similar in terms of age and PSA level, and although median estimated prostate weight was identical in left lateral position $(40 \mathrm{~g} \pm 10.6)$, modified lithotomy position $(40 \mathrm{~g} \pm 12.6)$, and standing-up position $(40 \mathrm{~g} \pm 11.2)$, there was a significant difference between the standard deviations of estimate prostate weight in left lateral position, modified lithotomy position, and squatting down with elbows on the table $(45 \pm 8.1)$. Since the degree of prostatic enlargement is of more practical interest than the precise estimate of prostate volume (11), small differences between the groups may not be clinically significant.

Strengths of our study include that a single examiner performed all DRE per site. Patients were questioned about their preferences before examination, avoiding bias, and they were divided in groups according to their choices. Limitations to this study include a small survey response rate $(27.7 \%)$. However, the proportion between the different positioning preferences remained fairly the same throughout data collection. Randomization through patients' and examiners' preferences is not a recommended method for research purposes. Standard methods of randomization should be used in future studies. Another limitation was the relatively small sample size in each subgroup, especially for the squatting down with elbows on the table. A significantly larger sample of patients would be necessary to evaluate the rate of prostate cancer detection.

\section{CONCLUSIONS}

The preferred position reported by Brazilian Urologists to perform DRE of the prostate is the modified lithotomy position, while our patients prefer or think it is less embarrassing to receive DRE in left lateral position.

Results of DRE in the different positions evaluated demonstrate a faster examination time in the standing-up position, and similar prostate asymmetry rate, positive DRE rate, and incomplete palpation of the prostate rate. Pain, urinary urgency, and bowel urgency scores are also comparable between each position, except for squatting down with elbows on the table, which may show increased bowel urgency score. 


\section{CONFLICT OF INTEREST}

None declared.

\section{REFERENCES}

1. Guinan P, Bush I, Ray V, Vieth R, Rao R, Bhatti R: The accuracy of the rectal examination in the diagnosis of prostate carcinoma. N Engl J Med. 1980; 303: 499-503.

2. Hinman F. Rectal examination of prostate and seminal vesicles. In: Hinman F (ed.), The principles and practice of urology. Philadelphia and London, WB Saunders Company. 1935; pp. 303-5.

3. Lane TJ: A note on rectal examination of the adenomatous prostate. Ir J Med Sci. 1940; 15: 322-6.

4. Kontturi M: Symptoms and patient evaluation. In: Altwein JE (ed.), Benign prostatic hyperplasia: a diagnosis and treatment primer. New York, Merck \& CO, Inc. 1994; pp. 55-6.

5. Messina LE, Lima H, Andrade E, Pinto GA, Srougi M, Ortiz V: Estudo da preferência da posição que urologistas brasileiros utilizam para realizar o toque prostático. In: $30^{\circ}$ Congresso Brasileiro de Urologia, 2005, Brasília. Anais do Congresso. Rio de Janeiro: Press Graphic \& Publishing Ltd., 2005; vol 30, p. 19-9.

6. Furlan AB, Kato R, Vicentini F, Cury J, Antunes AA, Srougi M: Patient's reactions to digital rectal examination of the prostate. Int Braz J Urol. 2008; 34: 572-5; discussion 576.

7. Frank J, Thomas K, Oliver S, Andrews S, Choong $\mathrm{S}$, Taylor R, et al.: Couch or crouch? Examining the prostate: a randomized study comparing the knee-elbow and the left-lateral position. BJU Int. 2001; 87: 331-3.

8. Catalona WJ, Richie JP, Ahmann FR, Hudson MA, Scardino PT, Flanigan RC, et al.: Comparison of digital rectal examination and serum prostate specific antigen in the early detection of prostate cancer: results of a multicenter clinical trial of 6,630 men. J Urol. 1994; 151: 1283-90.

9. Romero FR, Romero AW, Brenny Filho T, Bark NM, Yamazaki DS, de Oliveira FC: Patients' perceptions of pain and discomfort during digital rectal exam for prostate cancer screening. Arch Esp Urol. 2008; 61: 850-4.

10. Kaygisiz O, Inal G, Tas M, Ugurlu O, Ozturk B, Adsan O: Can pain during digital rectal examina- tion help us to decide the necessity and the method of anesthesia for transrectal ultrasound guided prostate needle biopsy? Int Braz J Urol. 2007; 33: 470-3; discussion 474-6.

11. Loeb S, Han M, Roehl KA, Antenor JA, Catalona WJ: Accuracy of prostate weight estimation by digital rectal examination versus transrectal ultrasonography. J Urol. 2005; 173: 63-5.

Accepted after revision:

October 18, 2010

\author{
Correspondence address: \\ Dr. Frederico R. Romero \\ R. Emiliano Perneta, 653 / 41 \\ Curitiba, PR, 80420-080, Brazil \\ Fax: + $55413324-9088$ \\ E-mail: frederico.romero@gmail.com
}

\section{EDITORIAL COMMENT}

The need to undergo a Digital rectal examination (DRE) as part of the Prostate cancer screening continues to torment many patients. Despite many articles suggesting that it is unnecessary, DRE continues to have a role in the diagnosis and management of prostate cancer as the search for the perfect marker continues.

Romero et al. have reported on patient positioning during DRE of the prostate. They have analysed both the clinicians' and the patients' preference about undertaking/undergoing a DRE. It is no surprise that most patients prefer the left lateral position which is my experience in the United Kingdom as well as the Far East. This could be attributed to the ability to avoid eye contact in this position. Also, the foetal position is somewhat comforting. On the other hand, The North American Urologists tend to prefer the knee-elbow/bent over position which is quicker and less taxing on the mobilitychallenged.

An important finding in the study is the total completeness of the examination in the kneeelbow position. This suggests in a select group of 
patients such as the markedly obese, this position would be appropriate.

This article suggests findings which should enable urologist to undertake a better quality DRE especially from the patient perspective.

Dr. Joe Philip

Department of Urology

Leighton Hospital

Cheshire CW1 4QJ United Kingdom

E-mail: indianajoe@gmail.com practice we attempt to adopt to our patients needs and comfort in order to keep their compliance and cooperation, therefore the lateral position seems to be more popular while doing full abdomino-pelvic examination. However it is common to use a standing up position for annual prostate screening and therefore this data should be correlated to the status of the visit (first, routine annual or part of other urological investigated issues) and be explained to the examinee beforehead. One should realize that even a small, simple clinical issue might be so important for the well being of the patients, for his further cooperation that eventually will lead to a better diagnosis and early detection of treatable disease.

\section{REFERENCES}

various complaints. The patient's position should adopt according to their desire, comfort, physical condition and the indication concerned. The purpose of the study was to evaluate the preferred DRE position by the urologists and their patients, a comparison between four typical positions. The exam is important to the diagnosis of anorectal disorders and prostate palpation. It is considered as one of the physician basic skills (1). Without a DRE, patients would have their disease detected only by the presence as urinary symptoms and cancer would be more likely to have higher stage and less potentially curable (2). The quality and degree of the pain and discomfort is debatable among studies, encourage us to find the best clinical set up for patients acceptance and cooperation even in their inexperienced first visit (3-5). A previous attempt to investigate the tolerability and acceptance of the patients have been presented by Furlan et al., yet this new study sheds more light and adequate statistical power (3). As for this manuscript, one can consider retrieving data regarding doctor's finger diameter and length; it might influence the patients discomfort in specific positions.

Left or right lateral position seems to be mostly preferred by the patients while the modified lithotomy position is mostly preferred by the urologists. The main argument for the patients prefaration is decreased embarrassment. In our every day
1. van Driel MF, van Andel MV, ten Cate Hoedemaker HO, Wolf RF, Mensink HJ: Physical diagnosis--digital rectal examination. Ned Tijdschr Geneeskd. 2002; 146: 508-12. Erratum in: Ned Tijdschr Geneeskd. 2002; 146: 928.

2. Sutton MA, Gibbons RP, Correa RJ Jr.: Is deleting the digital rectal examination a good idea? West J Med. 1991; 155: 43-6.

3. Furlan AB, Kato R, Vicentini F, Cury J, Antunes AA, Srougi M: Patient's reactions to digital rectal examination of the prostate. Int Braz J Urol. 2008; 34: 572-5; discussion 576.

4. Romero FR, Romero AW, Brenny Filho T, Bark NM, Yamazaki DS, de Oliveira FC: Patients' perceptions of pain and discomfort during digital rectal exam for prostate cancer screening. Arch Esp Urol. 2008; 61: 850-4.

5. Romero FR, Romero KR, Brenny FT, Pilati R, Kulysz D, de Oliveira Júnior FC: Reasons why patients reject digital rectal examination when screening for prostate cancer. Arch Esp Urol. 2008; 61: 759-65.

Dr.Avraham Shtricker Department of Urologic Surgery The Edith Wolfson Medical Center The Sackler School of Medicine Tel Aviv University, Israel E-mail:shtrickeravi@hotmail.com 


\section{EDITORIAL COMMENT}

The authors evaluated the preferred position for digital rectal examination (DRE) used by Brazilian Urologists and patients. They also analyzed results and tolerability of different positions. DRE should be part of urological evaluation; PSA does not reduce the value of DRE. This kind of papers emphasizes the importance of DRE.

In this study preferred position is written and discussed well, study gives information about pain score and even urinary and bowel urgency score. However modified lithotomy position was preferred by Brazilian Urologists, different positions could be selected in different country with the similar pain and discomfort rate. This study could guide the clinician to select the position; for exam-

\section{REFERENCES}

1. Furlan AB, Kato R, Vicentini F, Cury J, Antunes AA, Srougi M: Patient's reactions to digital rectal examination of the prostate. Int Braz J Urol. 2008; 34: 572-5; discussion 576. ple standing-up position could be selected in busy clinics with lower total DRE time.

Mean pain score of DRE is 5.0, this high pain score should not discourage the urologist to perform DRE since pain score was lower in previous literatures $(1,2)$ as written in discussion.

Limited patients' number of group 4 and position selection criteria with examiners' and patients' preferences are deficiency for comparing the DRE pain and tolerability between groups as written in discussion. This analyze could not give us definite judgment however it is well written paper for patient and urologist selection with pain and tolerability for whole groups and it should have been the essence of the study.

2. Kaygisiz O, Inal G, Tas M, Ugurlu O, Ozturk B, Adsan $\mathrm{O}$ : Can pain during digital rectal examination help us to decide the necessity and the method of anesthesia for transrectal ultrasound guided prostate needle biopsy? Int Braz J Urol. 2007; 33: 470-3; discussion 474-6.

Dr. Onur KAYGISIZ Urology Department Yalova State Hospital Yalova, TURKEY E-mail: onurkygsz@yahoo.com 